\title{
A second generation genetic map of the bumblebee Bombus terrestris (Linnaeus, 1758) reveals slow genome and chromosome evolution in the Apidae
}

Eckart Stolle $^{1 *}$, Lena Wilfert ${ }^{2,4}$, Regula Schmid-Hempel ${ }^{2}$, Paul Schmid-Hempel ${ }^{2}$, Michael Kube ${ }^{3}$, Richard Reinhardt ${ }^{3,5}$, Robin FA Moritz ${ }^{1}$

\begin{abstract}
Background: The bumblebee Bombus terrestris is an ecologically and economically important pollinator and has become an important biological model system. To study fundamental evolutionary questions at the genomic level, a high resolution genetic linkage map is an essential tool for analyses ranging from quantitative trait loci (QTL) mapping to genome assembly and comparative genomics. We here present a saturated linkage map and match it with the Apis mellifera genome using homologous markers. This genome-wide comparison allows insights into structural conservations and rearrangements and thus the evolution on a chromosomal level.

Results: The high density linkage map covers 93\% of the B. terrestris genome on 18 linkage groups (LGs) and has a length of 2'047 cM with an average marker distance of $4.02 \mathrm{cM}$. Based on a genome size of $\sim 430 \mathrm{Mb}$, the recombination rate estimate is $4.76 \mathrm{cM} / \mathrm{Mb}$. Sequence homologies of 242 homologous markers allowed to match 15 B. terrestris with A. mellifera LGs, five of them as composites. Comparing marker orders between both genomes we detect over $14 \%$ of the genome to be organized in synteny and $21 \%$ in rearranged blocks on the same homologous LG.

Conclusions: This study demonstrates that, despite the very high recombination rates of both $A$. mellifera and $B$. terrestris and a long divergence time of about 100 million years, the genomes' genetic architecture is highly conserved. This reflects a slow genome evolution in these bees. We show that data on genome organization and conserved molecular markers can be used as a powerful tool for comparative genomics and evolutionary studies, opening up new avenues of research in the Apidae.
\end{abstract}

\section{Background}

The buff-tailed bumblebee Bombus terrestris is a key pollinator for crops and wild flowering plants as well as a model system in various disciplines of biological research. This includes studies on population genetics, mating biology, sexual selection, caste determination, social behavior, host-parasite interactions, immunology and plant-pollinator interactions [1-11]. In addition, colonies of $B$. terrestris are commercially produced in large numbers in Europe for pollination of greenhouse

\footnotetext{
* Correspondence: eckart.stolle@zoologie.uni-halle.de

${ }^{1}$ Institut für Biologie, Martin-Luther-Universität Halle-Wittenberg, Hoher Weg 4, D-06099 Halle (Saale), Germany

Full list of author information is available at the end of the article
}

crops [1]. Accordingly, many genomic resources have been developed for this species such as molecular markers [12-15], genetic linkage maps [16,17] and BACand EST-libraries $[18,19]$.

With the advance of genome sequencing techniques $B$. terrestris is about to evolve into an important Hymenopteran genetic model species in addition to the honeybee, Apis mellifera and the parasitic wasp Nasonia spp. Since the bumblebee is phylogenetically very similar to $A$. mellifera with its fully sequenced genome, a genomic comparison between the two species is particularly rewarding for understanding genome evolution in social bees. The genome of $A$. mellifera revealed several exceptional traits including an extremely high recombination 
rate, a very high AT-content, the lack of retrotransposons, and a high density of simple-sequence-repeats (SSR/microsatellites) [20]. The evolution of these extraordinary genome characteristics is unclear. A comparison with the bumblebee genome might therefore reveal common patterns resulting from the phylogenetically close relationship, but also differences due to different social colony structures and ecologies of honeybees and bumblebees.

High resolution genetic maps are powerful tools to study genomic organization [21,22]. Moreover, such maps greatly facilitate genome assembly for full genome sequencing [23]. Whereas most of the first genetic maps were based on markers like RAPD, AFLP, isozymes or mutant phenotypes, linkage maps are now increasingly constructed with polymorphic simple sequence repeats (SSR, microsatellites) or single nucleotide polymorphisms (SNP) [23-35]. Since these markers also include sequence information of potentially conserved flanking regions, they allow for anchoring genome assemblies and for comparisons among species [24,28,32,35-37].

For $B$. terrestris two basic linkage maps are available: one map based on RAPD and SSR markers [16] and another map with AFLP and SSR markers $[17,38]$. However, in both maps the coverage and marker density was insufficient to explicitly detect all known 18 chromosomes of this species' haploid set [39]. Moreover, these maps could not be used for genomic comparisons between the honeybee and the bumblebee, because most markers were either RAPDs or AFLPs, which do not provide any sequence information.

In this paper we construct a dense and saturated genetic (meiotic) linkage map for the bumblebee $B$. terrestris using recently published SSR markers [15] as well as novel SSRs created from BAC-end sequences. Based on this second generation linkage map and sequence homologies of microsatellite-flanking regions, we compare the genetic maps of $B$. terrestris and A. mellifera to identify homologous chromosomes, conserved synteny blocks and rearrangements. These can be used to study chromosome and genome evolution as well as QTL synteny among species.

\section{Results}

\section{SSR markers}

A screen of the BAC library [19] yielded 4'593 SSRs with motifs of 1-6 bp in length of which 2'573 (56\%) were redundant or had too short sequences that were flanking the repeat motif. For the remaining 2'020 loci, a total of 960 primer pairs were tested for amplification products. 910 of those (95\%) yielded PCR products and were screened for polymorphisms in B. terrestris. 586 primer pairs $(64.4 \%)$ showed two or more alleles of which 564 were tested for polymorphism in the mapping population "BBM1" [17], a subset of 300 loci by using fluorescent labels, 264 loci by using unlabeled primers. This resulted in a total of 306 informative loci. The 123 SSR loci published in ref. [15] yielded 56 additional polymorphic loci in the population BBM1 and further three novel loci were developed as described in [15]. A screen of 2'304 A. mellifera SSR markers [23,40-42] yielded 15 loci that were polymorphic in BBM1. (Additional file 1). Finally 274 SSRs were successfully or sufficiently genotyped.

\section{Map}

To construct the new linkage map, we used the raw data (207 AFLPs, 39 SSRs) from the mapping population BBM1 (which was used for the core linkage map [17]) plus another 46 SSRs from ref. [15] and 209 SSRs derived from the BAC library (Additional file 2). Additionally three novel markers and 15 Apis mellifera [23,40-42] SSR markers were mapped (Table 1, Additional file 2). Four AFLPs remained unmapped. Although 75 markers showed segregation distortion, they were nevertheless included because their exclusion did not alter the map (Table 1).

Processing all available genotype data in JoinMap4 [43] yielded 18 linkage groups (LGs) all of which were well supported by LOD scores of 8.0 or higher (Additional file 3 ). The 18 LGs, which most likely represent the 18 haploid chromosomes [39], range in recombination size from 51.01 to $171.7 \mathrm{cM}$ containing 8 to 38 markers (Table 1). The shortest one, LG B18, contains only five AFLP and three SSR markers, the longest LG (B06) has 35 markers. The length of a LG was correlated with the number of markers per linkage group (Pearson $r=0.71768, p<0.05)$. The average marker distance ranges from 2.54 cM (LG B03) to $8.31 \mathrm{cM}$ (LG B17) with an average of $4.02 \mathrm{cM}( \pm 1.42 \mathrm{cM} \mathrm{SD})$.

This map contains a total 516 markers and spans a total of 1'902.21 cM (Additional file 2, Additional file 3). This is an increase of $271.21 \mathrm{cM}(16.62 \%)$ compared to [17] (1'630.9 cM, reanalyzed with JoinMap4 [43]). To correct for the missing chromosome ends, which cannot be mapped since there are no flanking markers, the length of each LG was adjusted by adding double its average marker distance to the value calculated by JoinMap [44]. This resulted in a corrected map length of 2'047.09 cM (Table 1). Hence the genome coverage of the present map is estimated to be $92.92 \%$.

Based on the function $\mathrm{c}=1-\mathrm{e}^{-2 \mathrm{md} / \mathrm{L}}$ given in ref. [45] where $\mathrm{c}$ is the proportion of the genome within $\mathrm{d} c \mathrm{c}$ distance to a marker, $\mathrm{L}$ the estimated genome length and $\mathrm{m}$ the number of markers, $86.85 \%$ of the genome is located within the average marker distance of $4.02 \mathrm{cM}$ and $99.99 \%$ of the genome is located within $17.6 \mathrm{cM}$ distance to a marker. 
Table 1 Summary of the $B$. terrestris linkage map

\begin{tabular}{|c|c|c|c|c|c|c|}
\hline LG & length (cM) & markers (n) & distorted markers $(n)$ & avg. marker distance (cM) & corrected length (cM) & LG as in $[17,38]$ \\
\hline Bt.B01 & 121.01 & 38 & 5 & 3.18 & 127.38 & 8 \\
\hline Bt.B02 & 125.20 & 37 & 6 & 3.38 & 131.97 & 1 \\
\hline Bt.B03 & 96.35 & 38 & 1 & 2.54 & 101.42 & 4 \\
\hline Bt.B04 & 80.66 & 20 & 1 & 4.03 & 88.73 & 13, BB1_18 \\
\hline Bt.B05 & 102.84 & 30 & 5 & 3.43 & 109.69 & 11 \\
\hline Bt.B06 & 171.70 & 35 & 6 & 4.91 & 181.51 & BB1_15 \\
\hline Bt.B07 & 161.43 & 33 & 0 & 4.89 & 171.21 & 9 \\
\hline Bt.B08 & 91.64 & 30 & 5 & 3.05 & 97.75 & 2 \\
\hline Bt.B09 & 109.48 & 32 & 6 & 3.42 & 116.32 & 5 \\
\hline Bt.B10 & 126.46 & 35 & 9 & 3.61 & 133.69 & 12 \\
\hline Bt.B11 & 116.30 & 35 & 2 & 3.32 & 122.94 & 7 \\
\hline Bt.B12 & 111.39 & 34 & 3 & 3.28 & 117.94 & 3 \\
\hline Bt.B13 & 105.74 & 31 & 5 & 3.41 & 112.56 & 6 \\
\hline Bt.B14 & 73.44 & 21 & 4 & 3.50 & 80.43 & BB1_16 \\
\hline Bt.B15 & 96.55 & 33 & 13 & 2.93 & 102.40 & 10 \\
\hline Bt.B16 & 77.87 & 16 & 1 & 4.87 & 87.61 & BB1_17, BB1_20 \\
\hline Bt.B17 & 83.14 & 10 & 2 & 8.31 & 99.77 & 14 \\
\hline Bt.B18 & 51.01 & 8 & 1 & 6.38 & 63.77 & BB1_19 \\
\hline$\Sigma / \varnothing$ & 1902.21 & 516 & 75 & $4.02 \pm 1.42$ & 2047.09 & \\
\hline
\end{tabular}

For each Linkage Group (LG) the length in $\mathrm{CM}(\Sigma)$, the number of markers (SSR and AFLP) mapped on this LG $(\Sigma)$, the number of markers showing segregation distortion $(\Sigma)$, the average distance between two markers in $\mathrm{CM}$, the length in $\mathrm{CM}$ of the LG after correction for chromosome ends $(\Sigma)$, and the corresponding LG in $[17,38]$ are given. At the bottom the sums and the average marker distance \pm standard deviation is given, respectively.

\section{Genome size and recombination rate}

The genome size of the bumblebee $B$. terrestris previously was measured by flow cytometry $[16,17]$. The first measurement [16] was based on a staining method biased towards the AT portion of the genome, hence a correction is needed. The genomic AT-content of B. terrestris was estimated to be $61 \%$ by using $8.5 \mathrm{Mb}$ nonredundant sequences (data not shown) from the BAC library, representing about $1.98 \%$ of the genome. The honeybee AT-content is $67.3 \%$ [46], 6.3\% higher than the bumblebee. Consequently the DNA content $(0.27$ pg) as measured by ref. $[16,47]$ was corrected leading to an increase of the ratio (B. terrestris/A. mellifera DNA content) from 1.54 to 1.653 . Thus the genome size of the bumblebee Bombus terrestris was estimated to be $433 \mathrm{Mb}$.

A second estimate was obtained using the relation between genetic distance and physical distance for two markers from the two ends of a BAC clone [19]. The two markers SSR 0929 66j14 and SSR 924_66j14 are $0.494 \mathrm{cM}$ apart (Additional file 2). The average insert size of clones from the BAC library is $102.9 \mathrm{~kb}$, based on a selection of $\mathrm{n}=186$ clones which doesn't include this BAC clone [19]. Extrapolated onto the whole map, a genome size of about $426 \mathrm{Mb}$ is calculated. This nearly matches the previous estimate of $433 \mathrm{Mb}$. The average between both estimates is $430 \mathrm{Mb}$. However, preliminary data for the genome assembly of B. terrestris (Baylor College of Medicine Human Genome Sequencing Center, unpublished) give an additional estimate of about $250 \mathrm{Mb}$ for the size of the genome.

Using the length of this linkage map (2'047.09 cM), a recombination rate of $4.76 \mathrm{cM} / \mathrm{Mb}$ is calculated, based on a genome size of $430 \mathrm{Mb}$, and $8.19 \mathrm{cM} / \mathrm{Mb}$ based on a genome size of $250 \mathrm{Mb}$.

\section{Homology}

A search for homologous sequences in the A. mellifera genome for each mapped SSR marker yielded 242 homologous loci, with 15 being homologous with unassigned (unmapped) A. mellifera sequences. In 29 cases the $B$. terrestris sequence was homologous to a gene or a predicted gene in A. mellifera (Table 2, Additional file 1, Additional file 2).

A B. terrestris map containing only the loci homologous to the A. mellifera genome was constructed (Figure $1,2,3,4,5,6,7)$. By comparing both maps, it was possible to homologize 15 of the $18 \mathrm{~B}$. terrestris LGs with corresponding A. mellifera LGs (Table 2). Omitting homologues to unassigned $A$. mellifera sequences, 10 linkage groups could be precisely matched with 4 to 26 (mean 13.7) homologous loci. In case of LG B02 in $B$. terrestris, all homologous markers match LG 2 in $A$. mellifera. Five $B$. terrestris LGs were composites of parts 
Table 2 Matching linkage groups between $B$. terrestris and $A$. mellifera

\begin{tabular}{|c|c|c|c|c|c|c|c|c|c|c|c|c|c|c|c|c|c|c|}
\hline LG & $\begin{array}{c}A m . \\
\text { LG01 }\end{array}$ & $\begin{array}{c}\mathrm{Am} . \\
\mathrm{LG02}\end{array}$ & $\begin{array}{c}\mathrm{Am} . \\
\mathrm{LG03}\end{array}$ & $\begin{array}{c}A m . \\
\text { LG04 }\end{array}$ & $\begin{array}{c}A m . \\
\text { LG05 }\end{array}$ & $\begin{array}{c}A m . \\
\text { LG06 }\end{array}$ & $\begin{array}{c}\text { Am. } \\
\text { LG07 }\end{array}$ & $\begin{array}{c}A m . \\
\text { LG08 }\end{array}$ & $\begin{array}{c}\text { Am. } \\
\text { LG09 }\end{array}$ & $\begin{array}{c}A m . \\
\text { LG10 }\end{array}$ & $\begin{array}{c}A m . \\
\text { LG11 }\end{array}$ & $\begin{array}{c}A m . \\
\text { LG12 }\end{array}$ & $\begin{array}{c}A m . \\
\text { LG13 }\end{array}$ & $\begin{array}{c}\text { Am. } \\
\text { LG14 }\end{array}$ & $\begin{array}{c}A m . \\
\text { LG15 }\end{array}$ & $\begin{array}{c}\mathrm{Am} . \\
\text { LG16 }\end{array}$ & $\begin{array}{c}A m . \\
\text { Un }\end{array}$ & $\bar{\Sigma}$ \\
\hline Bt.B01 & 26 & & & 1 & 1 & & & & & & & & & & & & & 28 \\
\hline Bt.B02 & & 17 & & & & & & & & & & & & & & & 2 & 19 \\
\hline Bt.B03 & 1 & & 12 & & & & & 1 & & 1 & & & & & & 1 & 1 & 17 \\
\hline Bt.B04 & & & 1 & 4 & & & & & & & & & & & & & & 5 \\
\hline Bt.B05 & & & & & 19 & & 1 & & & & & & & & & & & 20 \\
\hline Bt.B06 & 2 & & 1 & & & 14 & & & & & & 1 & & & & & 1 & 19 \\
\hline Bt.B07 & 1 & & & & & & 3 & & & & 2 & & & & & & 2 & $\overline{8}$ \\
\hline Bt.B08 & & & & & & & 4 & 7 & & & & & & & & & 1 & 12 \\
\hline Bt.B09 & 4 & & & & & 5 & & & 12 & & & & & & & & & 21 \\
\hline Bt.B10 & & & & & & & 1 & & & 8 & & & & & & & 1 & 10 \\
\hline Bt.B11 & 6 & & & & 1 & & & & & & 7 & & & & & & 2 & 16 \\
\hline Bt.B12 & & & & & & & & & & & & 8 & & & & 7 & 1 & $\overline{16}$ \\
\hline Bt.B13 & & & & & & 1 & & & & & & & 16 & & & & 2 & 19 \\
\hline Bt.B14 & & & & 1 & & & & & & & & & & 10 & & & & $\overline{11}$ \\
\hline Bt.B15 & & & & & & & 1 & & & & & 7 & & & 6 & & & $\overline{14}$ \\
\hline Bt.B16 & 1 & & & & & & & 2 & & & & & & & & & 2 & 5 \\
\hline Bt.B17 & & & & & 1 & & & & & & & & & & & & & 1 \\
\hline Bt.B18 & & & & & & & & & & & & & & & & 1 & & 1 \\
\hline$\Sigma$ & 41 & 17 & 14 & 6 & 22 & 20 & 10 & 10 & 12 & 9 & 9 & 16 & 16 & 10 & 6 & 9 & 15 & 242 \\
\hline
\end{tabular}

The numbers of markers homologous between Bombus terrestris and Apis mellifera are shown for each LG. Bold numbers indicate matching $B$. terrestris and A. mellifera LGs, as homologized LGs (the majority of the homologous markers is found in one LG) or as composites if a LG consists of a high proportion of markers homologous to more than one A. mellifera LG. Single markers or low numbers of markers are left normal.

homologous to two different $A$. mellifera LGs each. 35 homologous loci were mapped on A. mellifera LGs that were different from the homologized ones. The three small LGs B16, B17 and B18 consist of too few homologous markers to assign them to A. mellifera LGs (Table 2, Additional file 1, Additional file 2).

Overall, there are many conserved chromosomal regions in both genomes. With 83 syntenic marker pairs from 15 bumblebee LGs spanning a total of $302.16 \mathrm{cM}$ in B. terrestris and corresponding to $689.80 \mathrm{cM}$ in $A$. mellifera. The distances between syntenic marker pairs ranged from 0.003 to $26.05 \mathrm{cM}$ and from 0.08 to 54.28 $\mathrm{cM}$ for $B$. terrestris and A. mellifera, respectively (Table 3, Additional file 4). In 18 cases three to six markers were conserved in sequential order. In total the syntenic regions account for $14.81 \%$ of the whole map, with the distribution among the different LGs being heterogenic. LGs B02 and B13 show the largest proportion of syntenic regions with $47.99 \%$ and $41.66 \%$, respectively. LGs B03 and B07 exhibit the lowest proportion with $1.16 \%$ and $0.3 \%$ syntenic regions, respectively (Table 3). The mean is $17.6 \%$.

Most chromosomal regions showed rearrangements in the spatial ordering of markers, but only within the same homologous LG. These cases reflect inversions or non-reciprocal translocations (chromosome mutations) (e.g. Figure 2: B02). While such regions cannot be precisely linked to physical positions on the map as there is no information about the exact locations of breakpoints, these markers are nevertheless located on the same chromosome. A total of 65 such blocks, which do not show an inter-chromosomal insertion, were found in $B$. terrestris and these account for $21.09 \%(431.76 \mathrm{cM})$ of the whole map length (Table 3, Additional file 5). The highest proportion of such homologous regions was found on the two LGs B05 and B14 with $50.63 \%$ and $45.89 \%$, respectively, whereas the two LGs B07 and B13 with 0 and $6.31 \%$, respectively, showed the lowest proportion: the mean proportion is $24.59 \%$ (Table 3 ).

Taking into account the syntenic and homologous rearranged proportions, a total of $35.9 \%$ of the whole map length is conserved between A. mellifera and B. terrestris. With more than 60\% the LGs B02 (73.99\%), B14 (68.24\%), and B05 (65.07\%) exhibit the highest degree of conservation, whereas the lowest degree was observed in LGs B07 (0.3\%), B03 (16.38\%) and B15 (17.79\%) (Table 3): the mean percentage of conservation was $42.19 \%$. Accordingly, a high percentage of the A. mellifera LGs are homologous but rearranged if compared to those of B. terrestris (Figure 1, 2, 3, 4, 5, 6, 7).

Inter-chromosomal (reciprocal) translocations of larger regions only occurred in five composite chromosomes 


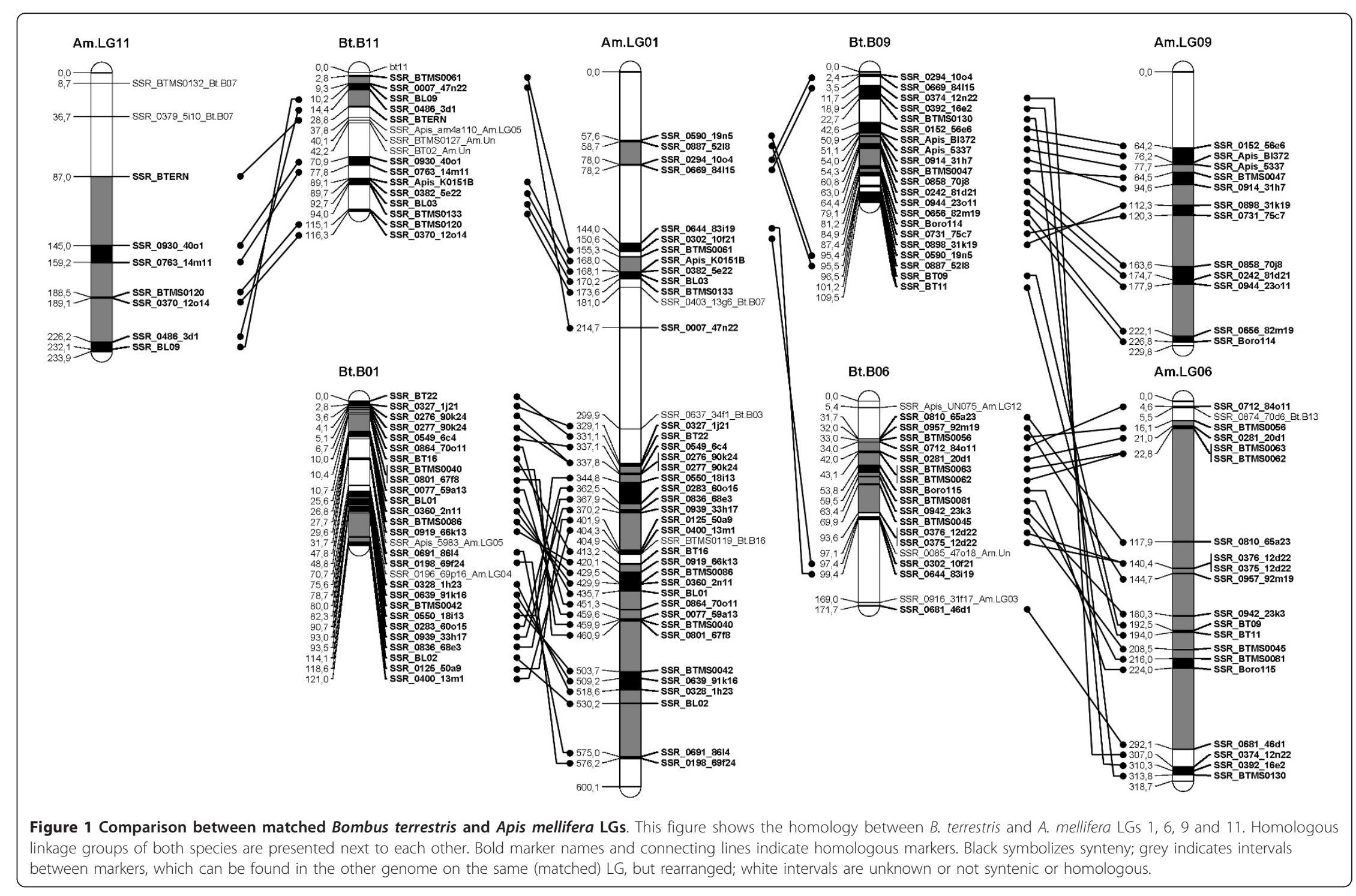




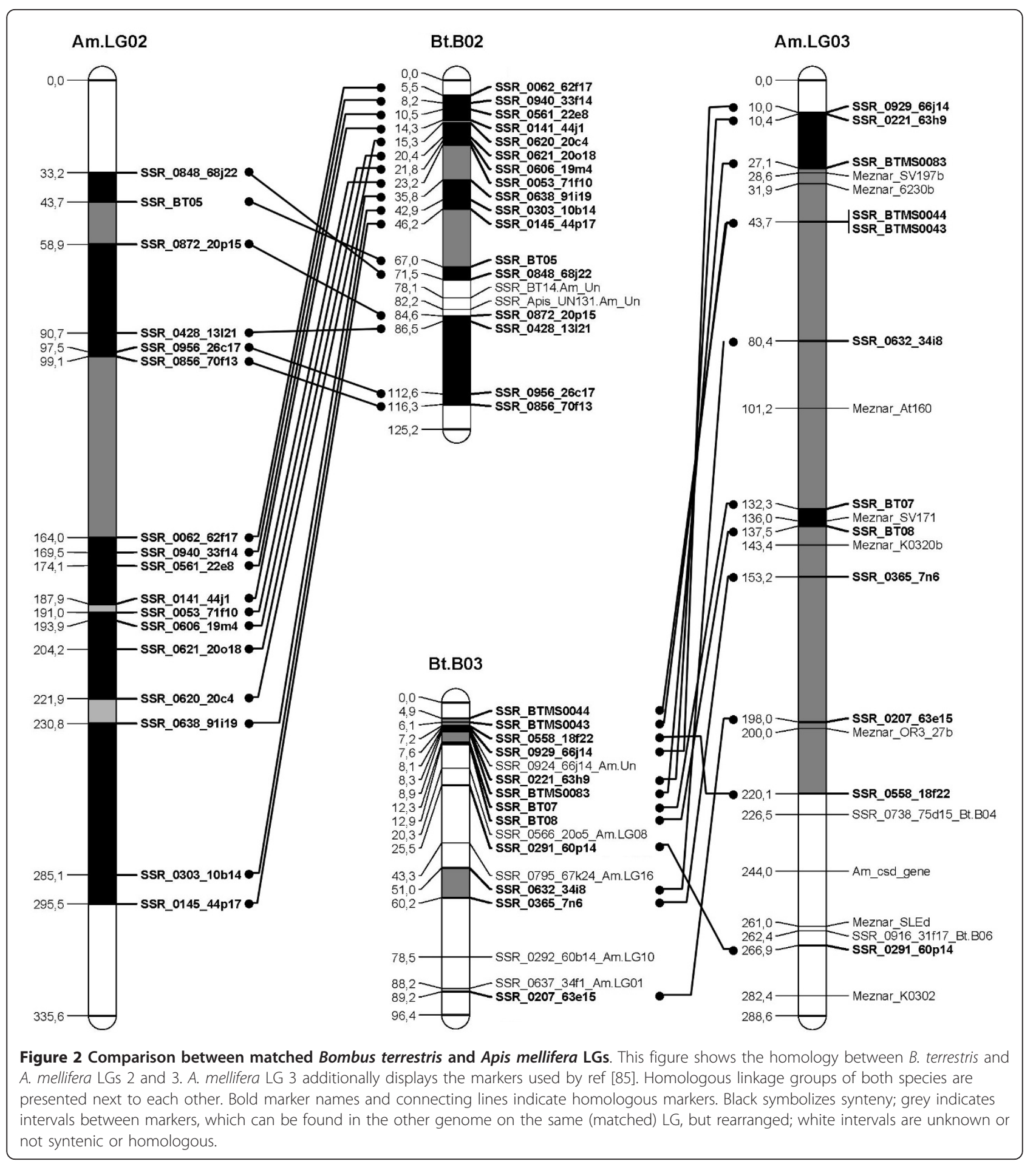

(see above). Small interchromosomal translocations (a single or double marker insertion) were only observed in 21 cases. Those markers were homologous to A. mellifera LGs except for 2, 9, 11, 13, 14 and 15 which had been "inserted" into B. terrestris LGs except in LG B02, B08 and B12 (Table 3, Additional file 2).

\section{Discussion}

We here present a second-generation linkage map of the bumblebee $B$. terrestris (Additional file 3). With 18 linkage groups spanning a total of 2'047.09 cM (Table 1) it matches the known number of the haploid chromosomal set $(n=18)$ [39]. Compared to the previous core 


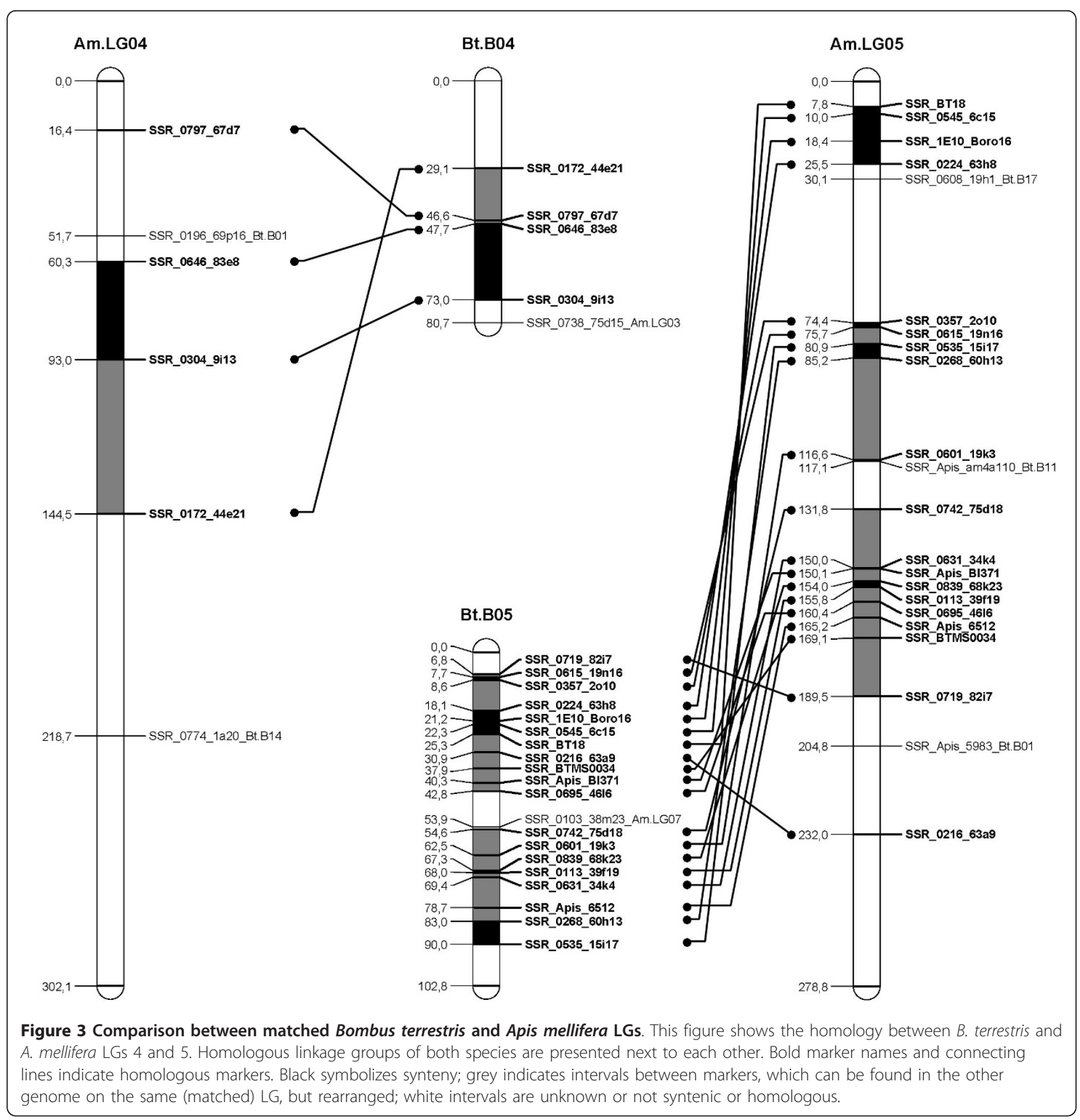

linkage map (BBM1, [17]) both the number of LGs ( $\mathrm{n}=$ $21)$ and the total map length $(2$ '221.8 cM) are considerably smaller. The shorter map length is a result of a different mapping algorithm compared to that of ref. [17] which used a maximum likelihood algorithm (Mapmaker [48]). The Mapmaker procedure per se assumes no crossover interference causing map inflation whereas the regression algorithm (JoinMap4 [43,49]) used in this study does account for interference hence producing much shorter maps although both algorithms use
Kosambi's mapping function [26,49-52]. Since crossover interference is common in the honeybee and other higher organisms [e.g. $[23,53,54]]$ it seems prudent to consider this mechanism for establishing the bumblebee map. This highlights the importance of choosing a appropriate mapping algorithm to generate comparable and more precise genetic maps. Although several markers showed segregation distortion, those markers were not excluded, since the algorithm $\left(\mathrm{G}^{2}\right.$-statistics for independence) of JoinMap is not affected by segregation 


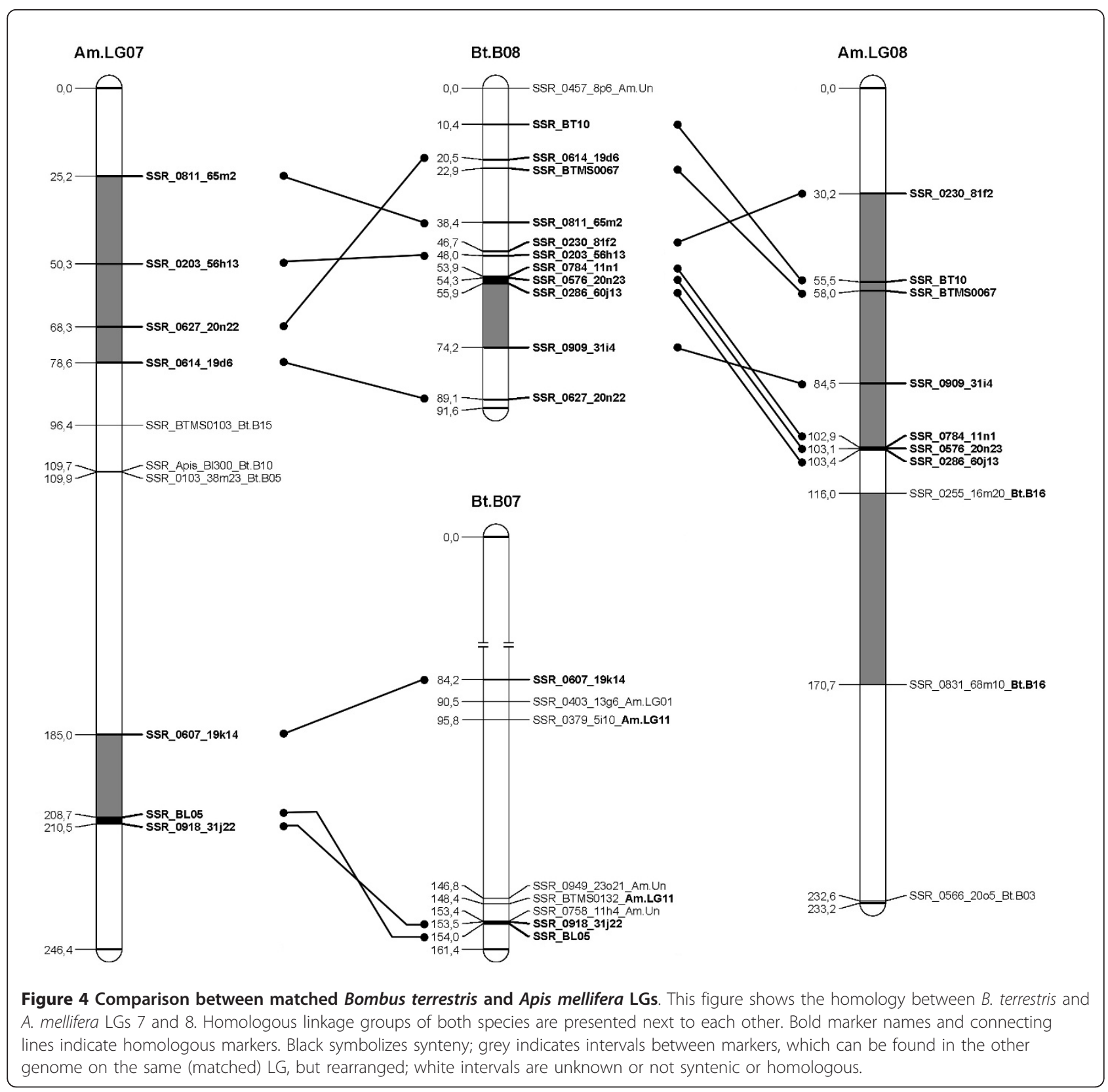

distortion [43]. In some case, the Segregation Distortion (meiotic drive) likely is caused by genotype gaps. But it can also have a biological background such as asymmetry of the meiosis (driving allele ends up in the ovocyte instead of in the polar bodies with a probability greater than one half) or can involve gamete destruction (postmeiotic mechanism, e.g. by a selfish segregation distorter genes as found in Drosophila, mouse and Tribolium). However, our data don't support further assumptions, since the distorted markers are distributed across almost all chromosomes (table 1) without showing a distinct pattern (Additional file 2).
The present 1'902.21 cM map (sizes not corrected for missing chromosome ends) contains 516 markers with an average distance of $4.02 \mathrm{cM}$ between markers. By reanalyzing the original data set used to create the core linkage map [17], we found that the map size was increased only by $16.6 \%$ (271.21 cM) by including 277 additional markers (map sizes not corrected for missing chromosome ends). The genome coverage $(92.92 \%)$ is much higher than the previous map's $81 \%$ [17]. 99.99\% of the genome is located within a distance of $17.6 \mathrm{cM}$ to a marker. The current map is thus nearly saturated and thus a valuable tool for further QTL mapping studies [2,3]. 


\section{Bt.B10}

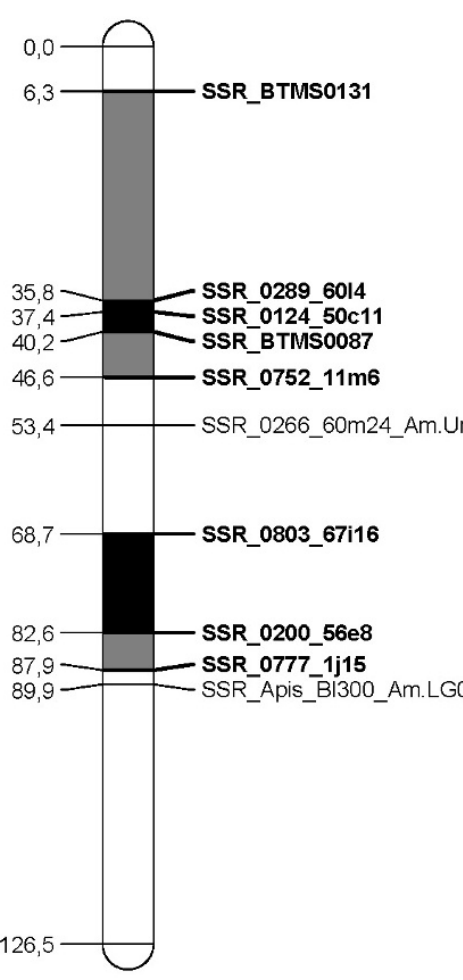

Am.LG10

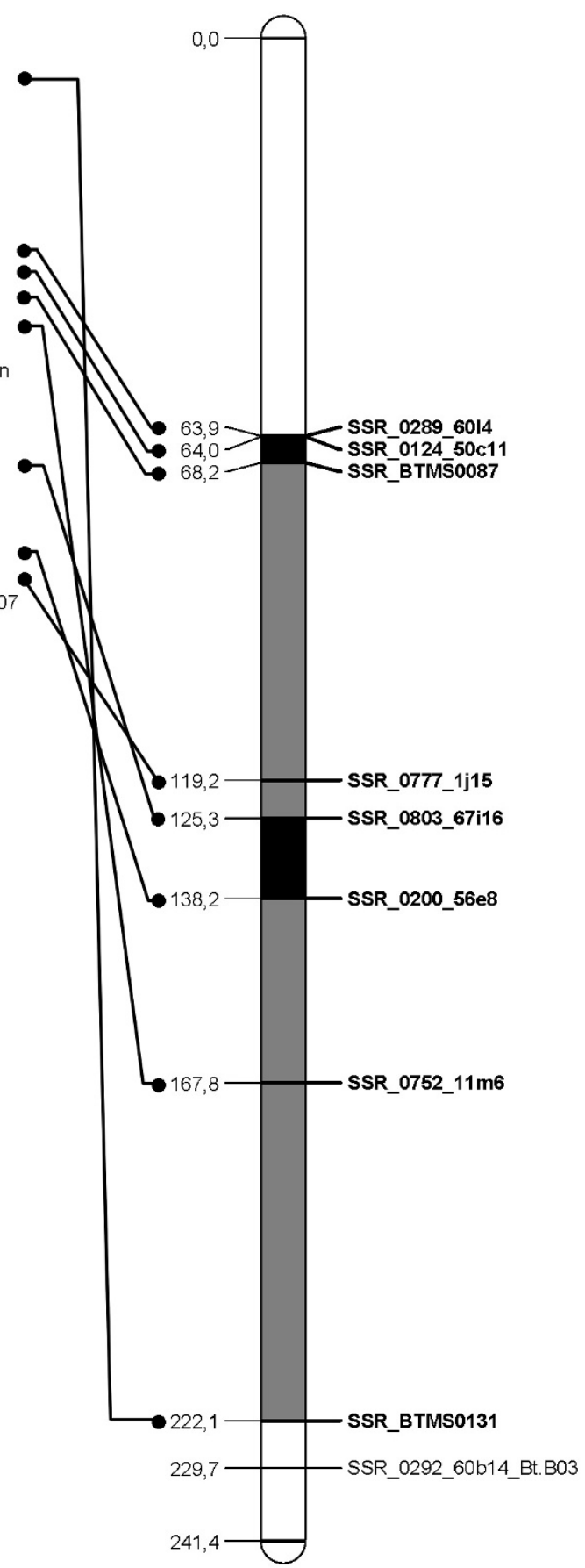

Figure 5 Comparison between matched Bombus terrestris and Apis mellifera LGs. This figure shows the homology between $B$. terrestris and A. mellifera LGs 10. Homologous linkage groups of both species are presented next to each other. Bold marker names and connecting lines indicate homologous markers. Black symbolizes synteny; grey indicates intervals between markers, which can be found in the other genome on the same (matched) $L G$, but rearranged; white intervals are unknown or not syntenic or homologous. 


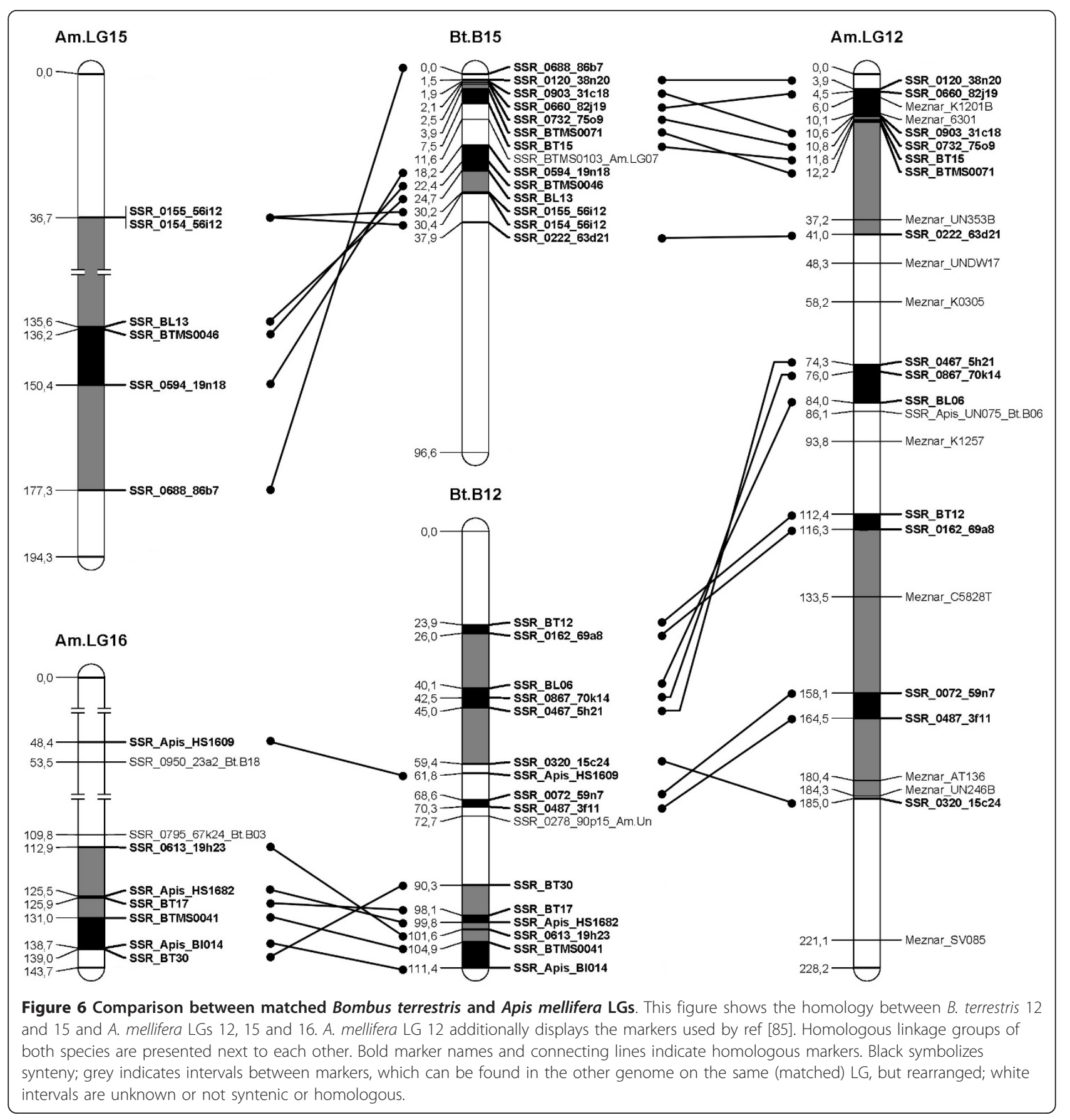

The two prior estimates for physical genome size were both based on flow cytometry and muscle cells, but differed substantially. Ref. [16] estimated a genome size of $274 \mathrm{Mb}$ whereas ref. [17] reported an estimate of 625 $\mathrm{Mb}$. The staining method used by [16] is typically biased towards the AT content of the genome [55-58] and hence may have lead to underestimating the genome size of $B$. terrestris because the $61 \%$ AT content is only $6.3 \%$ less than that of $A$. mellifera [46]. By correcting for the AT bias the $B$. terrestris genome size is estimated to be $433 \mathrm{Mb}$, very similar to the estimate of $426.41 \mathrm{Mb}$ in this study derived from the relation of the measured genetic and known physical distance between two neighboring markers. Theses concurring measurements lead us to the conclusion that the genome size reported by ref. [17] was overestimated. There is a further estimate of about $250 \mathrm{Mb}$ based on preliminary data for the $B$. terrestris genome assembly (Baylor College of Medicine 


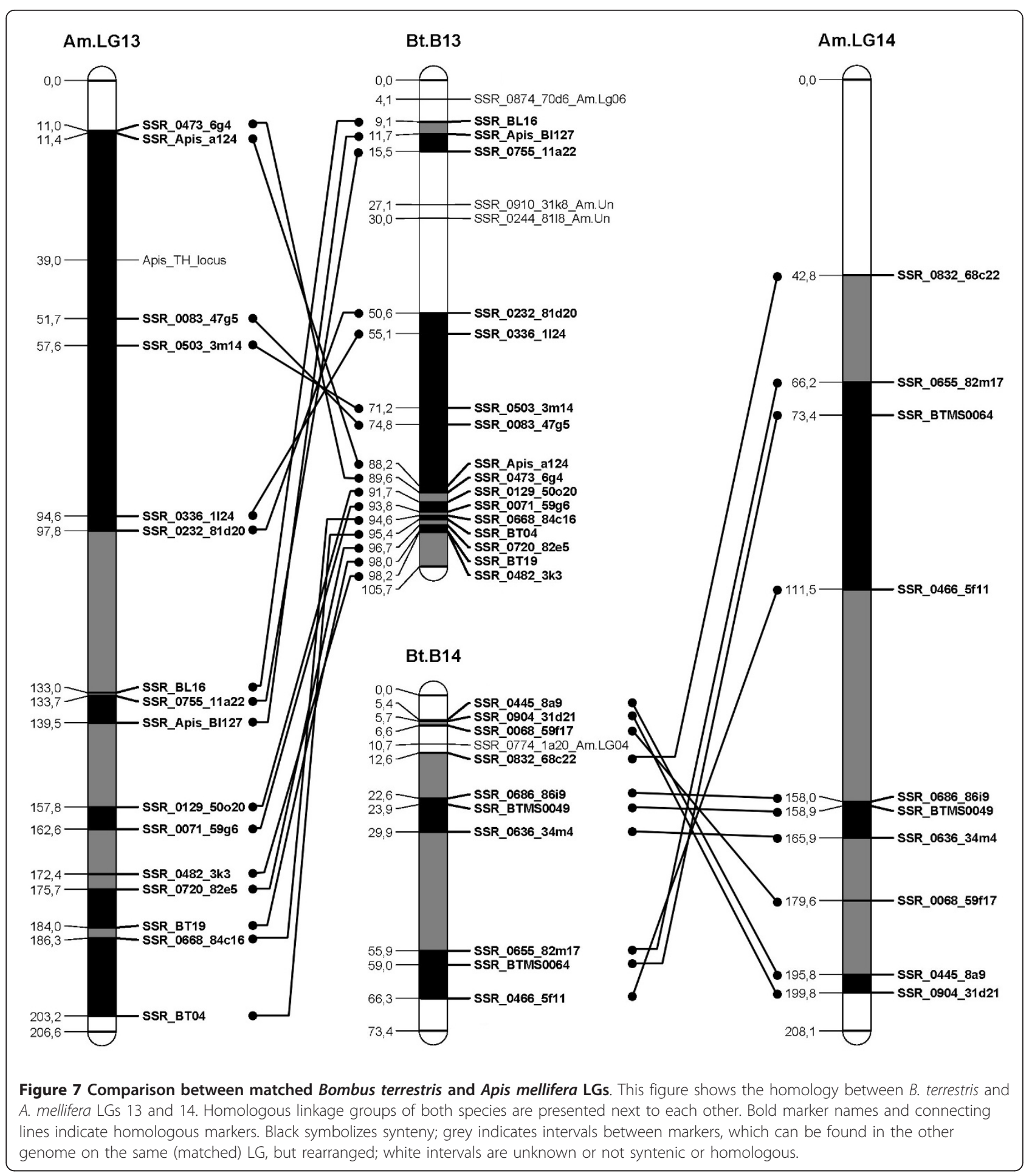

Human Genome Sequencing Center, unpublished), but this need to be verified.

Given a physical genome size of $430 \mathrm{Mb}$, the estimated recombination density of $4.76 \mathrm{cM} / \mathrm{Mb}$ for $B$. terrestris is slightly higher than the $4.42 \mathrm{cM} / \mathrm{Mb}$ previously published [17]. Although this recombination rate is much less than that of the honeybee genome $(15.7 \mathrm{cM} / \mathrm{Mb}$ [46]) it is still a high value compared to other eukaryotic organisms (Vertebrata $1.37 \mathrm{cM} / \mathrm{Mb}$, Insecta excl. Hymenoptera $2.69 \mathrm{cM} /$ $\mathrm{Mb},[46,59])$. This supports the idea that a high genomic recombination rate may be positively correlated with other genomic traits such as AT content, as shown for several 
Table 3 Summary of the positional information of homologous markers compared between Bombus terrestris and Apis mellifer $a$

\begin{tabular}{|c|c|c|c|c|c|c|c|c|c|c|}
\hline LG & $\begin{array}{l}\text { synteny } \\
\text { (n) }\end{array}$ & $\begin{array}{l}\text { synteny } \\
\text { (cM): B.t. }\end{array}$ & $\begin{array}{l}\text { synteny } \\
\text { (cM): A.m. }\end{array}$ & $\begin{array}{l}\text { synteny (cM): } \\
\text { ratio B.t./A.m. }\end{array}$ & $\begin{array}{l}\text { synteny } \\
\text { (\%): B.t. }\end{array}$ & $\begin{array}{c}\text { homology } \\
\text { (n) }\end{array}$ & $\begin{array}{l}\text { homology } \\
\text { (cM): B. t. }\end{array}$ & $\begin{array}{l}\text { homology } \\
\text { (\%): B.t. }\end{array}$ & $\begin{array}{l}\text { inserts } \\
(n)\end{array}$ & $\begin{array}{c}\text { synteny }+ \\
\text { homology (\%): } \\
\text { B.t. }\end{array}$ \\
\hline Bt.B01 & 12 & 24.692 & 57.9 & 0.43 & 19.38 & 10 & 51.283 & 40.26 & 2 & 59.65 \\
\hline Bt.B02 & 12 & 63.332 & 170.18 & 0.37 & 47.99 & 3 & 34.315 & 26.00 & 0 & 73.99 \\
\hline Bt.B03 & 2 & 1.179 & 22.03 & 0.05 & 1.16 & 4 & 15.43 & 15.21 & 4 & 16.38 \\
\hline Bt.B04 & 1 & 25.333 & 32.66 & 0.78 & 28.55 & 2 & 18.614 & 20.98 & 1 & 49.53 \\
\hline Bt.B05 & 6 & 15.837 & 25.25 & 0.63 & 14.44 & 11 & 55.54 & 50.63 & 1 & 65.07 \\
\hline Bt.B06 & 3 & 8.859 & 16.37 & 0.54 & 4.88 & 8 & 55.006 & 30.30 & 3 & 35.19 \\
\hline Bt.B07 & 1 & 0.506 & 1.74 & 0.29 & 0.30 & 0 & 0 & 0.00 & 1 & 0.30 \\
\hline Bt.B08 & 2 & 2.058 & 0.51 & 4.04 & 2.11 & 1 & 18.258 & 18.68 & 0 & 20.78 \\
\hline Bt.B09 & 11 & 33.858 & 95.42 & 0.35 & 29.11 & 4 & 27.785 & 23.89 & 4 & 52.99 \\
\hline Bt.B10 & 3 & 18.244 & 17.15 & 1.06 & 13.65 & 3 & 41.25 & 30.86 & 1 & 44.50 \\
\hline Bt.B11 & 6 & 17.201 & 26.36 & 0.65 & 13.99 & 2 & 20.886 & 16.99 & 1 & 30.98 \\
\hline Bt.B12 & 6 & 16.823 & 28.27 & 0.60 & 14.26 & 5 & 41.498 & 35.18 & 0 & 49.45 \\
\hline Bt.B13 & 9 & 46.896 & 122.42 & 0.38 & 41.66 & 5 & 7.1 & 6.31 & 1 & 47.97 \\
\hline Bt.B14 & 5 & 17.977 & 57.11 & 0.31 & 22.35 & 3 & 36.91 & 45.89 & 1 & 68.24 \\
\hline Bt.B15 & 4 & 10.327 & 21.29 & 0.49 & 10.08 & 4 & 7.885 & 7.70 & 1 & 17.78 \\
\hline Bt.B16 & 0 & 0 & 0 & 0 & 0 & 0 & 0 & 0 & 0 & 0.00 \\
\hline Bt.B17 & 0 & 0 & 0 & 0 & 0 & 0 & 0 & 0 & 0 & 0.00 \\
\hline Bt.B18 & 0 & 0 & 0 & 0 & 0 & 0 & 0 & 0 & 0 & 0.00 \\
\hline$\Sigma / \varnothing$ & $\Sigma 83$ & $\Sigma 303.029$ & $\Sigma 681.56$ & $\varnothing 0.45$ & ø 17.59 & $\Sigma 65$ & $\Sigma 429.815$ & ø 24.59 & $\Sigma 21$ & $ø 42.19$ \\
\hline
\end{tabular}

For each LG information on the number ( $\mathrm{n}$ ) of marker pairs (intervals/segments) is given, which is also present as a marker pair (interval/segment) in $A$. mellifera (synteny). The genetic length in $\mathrm{cM}$ of the synthenic intervals/segments in B. terrestris (B.t.) and the corresponding intervals/segments in A. mellifera (A.m.) and the ratio between both species is given. Furthermore, the proportion (\%) of synthenic marker intervals/segments of the total length of a $B$. terrestris LG is calculated. Likewise numbers of intervals/segments, their length (cM) and proportion (\%) of the total length in $B$. terrestris is listed for intervals/segments of markers present in B. terrestris, but rearranged (not paired) on the matching A. mellifera LG ("homology"), indicating intrachromosomal rearrangements. B. terrestris markers, which were found in a non-matching (see table 2) A. mellifera LG (interchromosomal rearrangement) are summed up as "inserts". The last column shows the proportion (\%) of synthenic plus homologous marker intervals/segments for each $B$. terrestris LG.

organism groups with the exception of mammals [46]. Alternatively, a high recombination rate might have evolved due to sex-restricted recombination (e.g. haplodiploid Hymenoptera) or may be related to sociality in insects as such [59]. Social Hymenoptera show a higher recombination rate (mean $10.27 \mathrm{cM} / \mathrm{Mb}, \mathrm{n}=4$ ) than nonsocial parasitoid Hymenoptera (mean $3.99 \mathrm{cM} / \mathrm{Mb}, \mathrm{n}=4$ ) $[46,59]$. Depending on the $B$. terrestris genome size in the final genome assembly, the recombination rate in the bumblebee might be significantly higher than estimated here. Based on a preliminary value of $250 \mathrm{Mb}$ a very high genome wide recombination rate of $8.19 \mathrm{cM} / \mathrm{Mb}$ is calculated making the relationships discussed above even more clear. However, the sample size for data on genomes from different taxonomic groups is still low, therefore a robust conclusion is not yet possible.

Using sequence similarities, it was possible to unambiguously match 15 linkage groups between $B$. terrestris and $A$. mellifera, of which five were composites consisting of partial homologous to two A. mellifera LGs (Table 2). A high proportion (21\%) of the genome showed homology in terms of markers present on the homologous LG, and $14.81 \%$ were identified as synteny blocks, segments with preserved marker order without disruption by rearrangements $[60,61]$. The genomic homology is most striking at the level of individual LGs. More than 40\% of LG B02 and B13 are syntenic. If synteny and rearranged blocks are added, on average a total of $42.19 \%$ of a LG is conserved. Three LGs even show a conservation of more than 65\% (Table 3).

This homology and synteny can be used to refer to previously mapped quantitative trait loci (QTLs) or genes in the honeybee (as shown above, Additional file 1). These loci may now serve as target candidate regions for the same traits in the bumblebee; hence, the map we present here can be a valuable tool for crossspecies genetic mapping. For example the thelytoky locus of A. mellifera [62] is located on chromosome nr. 13 , at $39 \mathrm{cM}$ between the syntenic marker pair SSR_Apis_a124 (11.4 cM) and SSR_0083_47g5 (51.66 cM) (Figure 7 , Additional file 2, 4). In B. terrestris this pair is located on LG B13 (88.2 cM and $74.8 \mathrm{cM}$, respectively). It is thus conceivable that the corresponding gene is located between the same markers in B. terrestris. 
A biologically important element is the sex locus (csd gene [63]), which is located on A. mellifera chromosome nr. 3 , at $243.95 \mathrm{cM}$. In the present new map there is unfortunately no syntenic marker pair surrounding this locus. The neighboring homologous markers are located on Bombus LG B04 and B06, whereas the remaining part of the chromosome is mostly homologous to LG B03 (Figure 1, 2, 3, Additional file 2, 4, 5). While this locus has already been mapped directly in B. terrestris too [16], it cannot be homologized with the honeybee, as the sex locus was linked only to RAPD markers. Hence, there is no unambiguous information for the location of the corresponding sex locus $c s d$ in B. terrestris. Its identification may require information on the whole genome sequence of the bumblebee [64-66].

Comparisons of genome architecture can provide insights into genome and chromosome evolution $[65,67,68]$. As we have shown, there is a high degree of homology between the genomes of $B$. terrestris and $A$. mellifera. On the other hand the divergence time between the bumblebees (tribe Bombini) and the honeybees (tribe Apini) has been roughly estimated based on fossil records and several phylogenetic or molecular systematic studies [69-74]. From this data, Bombini and Meliponini are considered to be sister groups, with the split of the Bombini (plus the Meliponini) and the Apini to have occurred 125 - 80 million years ago (mya) (mean 100 mya), coinciding with the Angiosperm radiation [75,76]. The genera Bombus and Apis are considered to have radiated much later into today's species diversity $[71,72]$. Despite an independent evolution of about 100 million years, large parts of the genome and even almost entire chromosomes are relatively conserved.

Other comparative genomic studies have revealed various degrees of conservation between genomes of species with different divergence times. In the genus Drosophila (age $\sim 40$ mya), for example extensive gene shuffling within the homologous chromosome arms between even moderately diverged genomes such as D. melanogaster and $D$. erecta (divergence $\sim 10$ mya [77]) is observed. The conservation of the genetic architecture between $D$. melanogaster and Rhagoletis pomonella (Diptera, Tephritidae) (divergence $\sim$ 50-55 mya) was high in chromosomes $\mathrm{X}$ and 3 , respectively, whereas $D$. melanogaster chromosome 2 is composed of regions homologous to all five R. pomonella LGs with many inter-chromosomal rearrangements [37]. In mammalian genomes, extensive shuffling of chromosomal regions between species (e.g. human, elephant, horse, hedgehog, cattle, cat, mouse) of phylogenetically different lineages, which split about 90 mya has been reported too [36,64,78-83]. Even within short evolutionary times (<40 mya) extensive genome reorganizations have been reported among the anthropoid Primates [84]. These exceed the differences between bumblebees and honeybees by far, although insects usually have much shorter generation lengths. Clearly many more rearrangements, both intra- and inter-chromosomal, have occurred among genomes of taxa with a similar divergence time as between $B$. terrestris and $A$. mellifera.

In light of these other studies, the large degree of homology between $B$. terrestris and $A$. mellifera is rather surprising. In fact, similar levels of homology as observed here are typical for very closely related species, such as mouse and rat (divergence 16 mya [64]) or with the example of the conserved marker order in chromosomes 3 and 12 of Apis mellifera and A. florea [85], which split 20-25 mya [71]. The high level of homology is furthermore surprising in light of the high genomewide recombination rate of both bee species, which clearly exceed the average recombination rate in insects or vertebrates [46,59].

Our findings suggest a very slow rate of genome and chromosomal evolution in these two bee species. This supports the previous conclusions that the honeybee genome evolved more slowly than that of the fruitfly or Anopheles mosquitoes [20]. Our new data and the conservation of marker order between two Apis species [85], suggests that the genome and chromosome evolution might be slow in the whole family Apinae.

Reasons for such a slow evolutionary rate at the genome level remain elusive. The relative lack of retrotransposons in A. mellifera [20] or the high density of simple-sequence-repeats (SSR, microsatellites) might be important factors. Sociality, which occurs in all four Apinae tribes, or haplodiploidy could also favor a slow genome evolution or vice versa. With the advance of next generation sequencing, it will clearly be only a matter of time until the whole genome sequence of Bombus terrestris and other bee species will be available. This will then allow us to conduct a comprehensive genomic comparison to unravel the ultimate evolutionary causes of the high genome conservation in social bees.

\section{Conclusions}

This report describes the construction of the first saturated linkage map for Bombus terrestris with 516 mapped markers. The genome coverage is 93\%. Based on homologies of microsatellite flanking sequences to the genome of Apis mellifera it was possible to match 15 linkage groups. A genome comparison revealed that about $15 \%$ of the genome is organized in syntenic blocks and $21 \%$ in rearranged regions on the same homologized linkage group. Inter-chromosomal rearrangements are less frequent. This high conservation of the genetic architecture is unexpected since both bee species exhibit a very high recombination rate and a long divergence time. This map will be an essential tool for QTL mapping, with the high degree of homology potentially 
allowing for cross species mapping in B. terrestris and $A$. mellifera.

\section{Methods \\ Mapping population \& DNA extraction}

A $B$. terrestris colony (BBM-1) was established as a phase-known mapping population with 577 male individuals [17]. It originated from a mated and hibernated queen from a wild catch in northwestern Switzerland. We used the same specimens (males) from this colony for this mapping study as well. DNA from the bumblebee individuals was extracted using the DNeasy Blood \& Tissue Kit (QIAGEN) following the manual.

\section{Genetic markers, PCR, genotyping}

End sequencing of a BAC-library [19] was carried out according to [86] and a screen for 1-5 bp simple sequence repeats (SSRs, microsatellites) was done using MISA [87]. Complete sequences containing the SSRs were checked against each other and already existing SSRs [12-15] for redundancy, employing a local BLAST search in BioEdit [88] or using the MAFFT alignment algorithm [89]. Primer pairs were designed with BatchPrimer3 [90], Primer3Plus [91] or manually for the resulting unique SSR loci. PCR was carried out at $50^{\circ} \mathrm{C}$, $55^{\circ} \mathrm{C}$ and $60^{\circ} \mathrm{C}$ using a TGradient thermocycler (Biometra) to optimize reaction conditions. Standard PCR reactions were performed in a total volume of $15 \mu \mathrm{L}(\sim 10$ ng DNA, $0.25 \mu \mathrm{L}$ of each primer $(10 \mu \mathrm{M}), 2.25 \mu \mathrm{L}$ of 10x reaction buffer $\left(160 \mathrm{mM}\left(\mathrm{NH}_{4}\right)_{2} \mathrm{SO}_{4}, 670 \mathrm{mM}\right.$ Tris$\mathrm{HCl}, 15 \mathrm{mM} \mathrm{MgCl} 2,0.1 \%$ Tween 20$), 0.13 \mu \mathrm{M}$ of a mix of each dNTP $(10 \mathrm{mM})$ and $0.3 \mathrm{U}$ Taq polymerase (GeneCraft), 3 min at $94^{\circ} \mathrm{C}, 37$ cycles of $45 \mathrm{~s}$ at $94^{\circ} \mathrm{C}$, $45 \mathrm{~s}$ at $50-60^{\circ} \mathrm{C}$ and $45 \mathrm{~s}$ at $72^{\circ} \mathrm{C}, 3 \mathrm{~min}$ at $72^{\circ} \mathrm{C}$ ). The PCR products were visualized on a $2 \%$ agarose gel stained with ethidium bromide and successfully amplifying loci were then checked for polymorphism in B. terrestris by performing a standard PCR containing a DNA pool from 11 B. terrestris queens or females (species identity was confirmed according to [92]) from Estonia (Tartu), France (Arles, Normandy), Hungary (Debrecen), Ireland (Belfast), Sweden (Tovetorp), Belgium (Zemst), Norway (Kalvøya), Austria (Vienna), laboratory colony (Koppert) and Germany (Halle) (5 ng each). The PCR products were run on a QIAxcel automatic capillary electrophoresis (QIAxcel DNA High Resolution Kit) and analyzed using the QIAxcel BioCalculator software (QIAGEN).

For a subset of polymorphic loci as well as the 123 microsatellite loci for $B$. terrestris recently published by ref. [15] fluorescent labeled primers (FAM, HEX or TET, Metabion) were used in multiplex standard PCR reactions (containing three primer pairs with a different fluorescent label and $20 \mathrm{ng}$ of a DNA pool from 10 males) to detect informative (dimorphic) loci in the mapping population. The PCR products were run on a MegaBace capillary sequencer and analyzed using the FragmentProfiler software. Additional loci were tested with unlabelled primer pairs in single locus PCR containing also $20 \mathrm{ng}$ of a DNA pool from 10 males of the mapping population and PCR products were run and analyzed on the QIAxcel system (see above).

The genotyping of 288 to 384 males from the mapping population was performed in multiplex PCRs with $2-10$ primer pairs depending on fragment size and fluorescent label. Multiplex PCRs with fluorescent labeled primer pairs were conducted using PCR Master Mix (Promega) and then run and analyzed on the MegaBace system (see above). Multiplex PCR's with unlabeled primer pairs were conducted using the standard PCR procedure (see above) and were run on the QIAxcel system (see above).

Worker-produced males were already detected and excluded by [17]. However, two more individuals with paternal alleles were detected and excluded from further analysis.

Preliminary information for an additional estimate of the genome size was obtained from Baylor College of Medicine Human Genome Sequencing Center (http:// www.hgsc.bcm.tmc.edu).

\section{Genotype analysis \& map construction}

For analysis of the genotypes the software JoinMap 4.0 [43] was used. The segregation was tested against the normal Mendelian expectation ration using a $\mathrm{Chi}^{2}$ test in order to detect Segregation Distortion. The software first detects linkage groups (LGs) based on the independence LOD (larger than 5) calculated for the recombination frequencies and the linkage phase is automatically determined using pairs with a LOD larger than 5 . The mapping was done phase-unknown using marker pair LOD scores of 5 or higher. Ref. [17] confirmed that in this system prior knowledge of linkage phase is not necessary for accurate genetic mapping (no difference between phase-known and phase-unknown mapping). Furthermore, the phase for some loci on each LG is known from [17], so the correct phase of each marker could be established. Then, for each LG, marker order and genetic distance were inferred by regression mapping using Kosambi's mapping function [50] to account for crossover interference. Three rounds were performed, using linkages with a recombination frequency smaller than 0.40 and a LOD larger than 1.0. After adding a single locus a "ripple" (test for all possible 3-point orders of consecutive markers to obtain the most likely order for every marker) was performed using linkage information from up to 10 neighboring markers to verify that the marker order found in previous analyses was correct. Maps were printed with the MapChart 2.2 software [93]. 


\section{Homology of SSR loci}

Using the available sequencing information for each mapped SSR (whole clone sequence containing the microsatellite, 337 to $961 \mathrm{bp}$ ) we performed a cross-species MegaBlast or alternatively BlastN search against the Apis mellifera genome (NCBI, Amel 4.0). Unique Blast hits with a homologous sequence larger than $30 \mathrm{bp}$, a score higher than 45 or a maximal identity of higher than $67 \%$ were used (two exceptions were made, where one of the characteristics had fallen below one of the thresholds). By plotting the genetic map [23] onto the physical map $[94,20]$, the genetic position on the respective A. mellifera linkage group could be estimated from the physical sequence homology (Blast hit). Next, individual maps for each linkage group of $B$. terrestris were plotted, only containing the homologous markers. Similarly all $A$. mellifera LGs were plotted again only using the homologous markers from the A. mellifera map. Both genomes were then compared side by side in MapChart2.2 [93].

\section{Additional material}

Additional file 1: SSR markers and Blast results. This table lists all used microsatellite markers. For novel SSR markers the GenBank accession numbers, the primer sequences with their annealing temperatures $(\mathrm{Ta})$, the repeat motif, the SSR type ( $\mathrm{c}$ - composite, $\mathrm{p}$ pure, number indicating the motif length), an approximate size range of the PCR fragment, an approximate number of alleles $\left(N_{a}\right)$, the BAC_ID (source of the repeat sequence) and (if applied) a fluorescent label are given. For all SSR markers the location (LG) in Bombus terrestris and Apis mellifera map, the origin/source is listed and the results of the Blast search against the Apis mellifera genome (Amel_4.0) as well as the Blast method are presented.

Additional file 2: Mapping data. For each mapped marker (AFLP and SSR) the genetic position on the LG, the distance (interval) to the next (following) marker and the genetic position within the A. mellifera genome (if a homologue was found) is given. Furthermore the linkage phase in the used mapping population is given. Significance ( $p$-value) of segregation distortion $\left(\mathrm{Chi}^{2}\right.$ test of allele frequencies for deviation from Mendelian segregation ratio) is indicated by stars $\left({ }^{*}: 0.1 ;{ }^{* *}: 0.05 ;{ }^{* * *}: 0.01\right.$; ${ }^{* * * *}: 0.005 ;{ }^{* * * *}: 0.001 ;{ }^{* * * * * *}: 0.0005 ;{ }^{* * * * * *}: 0.0001$ ).

Additional file 3: Bombus terrestris linkage map. This plot shows the Bombus terrestris linkage map with absolute marker positions and marker names for each linkage group.

Additional file 4: Synteny. This table shows syntenic marker pairs (intervals/segments) with their location ( $L G)$ and interval/segment length (CM) in B. terrestris (B.t.) and the corresponding interval/segment in A. mellifera (A.m.), as well as their ratio.

Additional file 5: Homology. This table shows marker pairs (intervals/ segments) from the B. terrestris (B.t.) map, of which both markers are located on a matching (see table 2) A. mellifera (A.m.) LG, but rearranged (not paired, hence no synteny). Their LG (B.t.) and interval/segment length (cM, B.t.) is given.

\section{Acknowledgements}

We thank Petra Leibe, Denise Kleber, Wanrong Zhou Bourke, Sandra Hangartner und Undine Zippler for technical assistance, Michel Solignac for providing many Apis-Primer, Alfred Beck for preparing the GenBank submissions, Ben Sadd for bioinformatic advice, Villu Soon, Keresztes Gabor,
Robert Paxton, Julia Stige, Jorgen Ravoet, Øistein Berg, Joseph Guedelph, Patrick Lhomme and Stephan Wolf for providing bumblebee samples, Gene Robinson, Kim Worley and Michael Lattorff for useful discussions. Preliminary sequence data was obtained from Baylor College of Medicine Human Genome Sequencing Center website at http://www.hgsc.bcm.tmc.edu [Funding was provided by the National Human Genome Research Institute, National Institutes of Health, U54 HG003273 (Richard Gibbs, PI)]. For financial support we thank the Max-Planck-Society (RR) and the German Science Foundation DFG (RFAM \& ES).

\section{Author details}

${ }^{1}$ Institut für Biologie, Martin-Luther-Universität Halle-Wittenberg, Hoher Weg 4, D-06099 Halle (Saale), Germany. ${ }^{2}$ Institute of Integrative Biology (IBZ), ETH Zürich, Universitätsstrasse 16, CH-8092 Zürich, Switzerland. ${ }^{3}$ Max Planck Institute for Molecular Genetics, Ihnestraße 63-73, D-14195 Berlin, Germany. ${ }^{4}$ Department of Genetics, University of Cambridge, Cambridge, CB2 3EH, UK. ${ }^{5}$ Genome Centre Cologne at MPI for Plant Breeding Research, Carl-vonLinné-Weg 10, D-50829 Köln, Germany.

\section{Authors' contributions}

ES carried out most of the experimental and conceptional work, microsatellite development, primer design and testing, genotyping and data analysis, map construction and drafting the manuscript. LW, RS and PS carried out the testing and genotyping of the $A$. mellifera primers, screened BAC sequences for microsatellites and provided original data and the mapping population from [17]. RR and MK carried out the BAC library end sequencing. RFAM was responsible for project conception, contributed drafting the manuscript and participated together with PS, RS and LW in design and coordination. All authors read and approved the final manuscript.

Received: 5 October 2010 Accepted: 19 January 2011

Published: 19 January 2011

\section{References}

1. Velthuis HHW, van Doorn A: A century of advances in bumblebee domestication and the economic and environmental aspects of its commercialization for pollination. Apidologie 2006, 37(4):421-451.

2. Wilfert L, Gadau J, Baer B, Schmid-Hempel P: Natural variation in the genetic architecture of a host-parasite interaction in the bumblebee Bombus terrestris. Molecular Ecology 2007, 16(6):1327-1339.

3. Wilfert L, Gadau J, Schmid-Hempel P: The genetic architecture of immune defense and reproduction in male Bombus terrestris bumblebees. Evolution 2007, 61(4):804-815.

4. Baer B, Schmid-Hempel P: Unexpected consequences of polyandry for parasitism and fitness in the bumblebee, Bombus terrestris. Evolution 2001, 55(8):1639-1643.

5. Baer B: Bumblebees as model organisms to study male sexual selection in social insects. Ecology and Sociobiology 2003, 54(6):521-533.

6. Pereboom JJM, Jordan WC, Sumner S, Hammond RL, Bourke AFG: Differential gene expression in queen-worker caste determination in bumble-bees. Proceedings of the Royal Society B-Biological Sciences 2005, 272(1568):1145-1152.

7. Kraus RB, Wolf S, Moritz RFA: The Male flight distance and population substructure in the bumblebee Bombus terrestris. Journal of Animal Ecology 2009, 78(1):247-252.

8. Plowright RC, Laverty TM: The ecology and sociobiology of the bumblebees. Review of Entomology 1984, 29:175-199.

9. Leadbeater $E$, Chittka L: The dynamics of social learning in an insect model, the bumblebee (Bombus terrestris). Behavioral Ecology and Sociobiology 2007, 61(11):1789-1796

10. Goulson D: Bumblebees - their behavior and Ecology. New York: Oxford University Press; 2003.

11. Schluns H, Sadd BM, Schmid-Hempel P, Crozier RH: Infection with the trypanosome Crithidia bombi and expression of immune-related genes in the bumblebee Bombus terrestris. Developmental and Comparative Immunology 2010, 34(7):705-709.

12. Estoup A, Solignac M, Harry M, Cornuet JM: Characterization of (GT) $\mathrm{n}$ and $(\mathrm{CT})_{\mathrm{n}}$ microsatellites in 2 insect species - Apis mellifera and Bombus terrestris. Nucleic Acids Research 1993, 21(6):1427-1431.

13. Estoup A, Tailliez C, Cornuet JM, Solignac M: Size homoplasy and mutational processes of interrupted microsatellites in 2 bee species, Apis 
mellifera and Bombus terrestris (Apidae). Molecular Biology and Evolution 1995, 12(6):1074-1084.

14. Reber-Funk C, Schmid-Hempel R, Schmid-Hempel P: Microsatellite loci for Bombus spp. Molecular Ecology Notes 2006, 6(1):83-86.

15. Stolle $E$, Rohde $M$, Vautrin D, Solignac M, Schmid-Hempel P, SchmidHempel R, Moritz RFA: Novel microsatellite DNA loci for Bombus terrestris (Linnaeus, 1758). Molecular Ecology Resources 2009, 9(5):1345-1352.

16. Gadau J, Gerloff CU, Kruger N, Chan H, Schmid-Hempel P, Wille A, Page RE: A linkage analysis of sex determination in Bombus terrestris (L.) (Hymenoptera : Apidae). Heredity 2001, 87:234-242.

17. Wilfert L, Gadau J, Schmid-Hempel P: A core linkage map of the bumblebee Bombus terrestris. Genome 2006, 49(10):1215-1226.

18. Sadd BM, Kube M, Klages S, Reinhardt R, Schmid-Hempel P: Analysis of a normalised expressed sequence tag (EST) library from a key pollinator, the bumblebee Bombus terrestris. BMC Genomics 2010, 11.

19. Wilfert L, Torres MM, Reber-Funk C, Schmid-Hempel R, Tomkins J, Gadau J, Schmid-Hempel P: Construction and characterization of a BAC-library for a key pollinator, the bumblebee Bombus terrestris L. Insectes Sociaux 2009, 56(1):44-48.

20. Weinstock GM, Robinson GE, Gibbs RA, Worley KC, Evans JD, Maleszka R, Robertson HM, Weaver DB, Beye M, Bork P, et al: Insights into social insects from the genome of the honeybee Apis mellifera. Nature 2006, 443(7114):931-949.

21. Erickson DL, Fenster CB, Stenoien HK, Price D: Quantitative trait locus analyses and the study of evolutionary process. Molecular Ecology 2004, 13(9):2505-2522

22. Wu R, Ma CX, Casella G: Statistical Genetics of Quantitative Traits Linkage, Maps, and QTL. Berlin, Heidelberg: Springer; 2007.

23. Solignac M, Mougel F, Vautrin D, Monnerot M, Cornuet JM: A thirdgeneration microsatellite-based linkage map of the honey bee, Apis mellifera, and its comparison with the sequence-based physical map. Genome Biology 2007, 8.

24. Brondani RPV, Williams ER, Brondani C, Grattapaglia D: A microsatellitebased consensus linkage map for species of Eucalyptus and a novel set of 230 microsatellite markers for the genus. Bmc Plant Biology 2006, 6.

25. Criscione CD, Valentim CLL, Hirai H, Loverde PT, Anderson TJC: Genomic linkage map of the human blood fluke Schistosoma mansoni. Genome Biology 2009, 10(6).

26. Hong YB, Chen XP, Liang XQ, Liu HY, Zhou GY, Li SX, Wen SJ, Holbrook CC, Guo BZ: A SSR-based composite genetic linkage map for the cultivated peanut (Arachis hypogaea L.) genome. Bmc Plant Biology 2010, 10.

27. Itoh T, Watanabe T, Ihara N, Mariani P, Beattie CW, Sugimoto Y, Takasuga A: A comprehensive radiation hybrid map of the bovine genome comprising 5593 loci. Genomics 2005, 85(4):413-424.

28. Kucuktas H, Wang SL, Li P, He CB, Xu P, Sha ZX, Liu H, Jiang YL, Baoprasertkul P, Somridhivej B, et al: Construction of Genetic Linkage Maps and Comparative Genome Analysis of Catfish Using GeneAssociated Markers. Genetics 2009, 181(4):1649-1660.

29. Lorenzen MD, Doyungan Z, Savard J, Snow K, Crumly LR, Shippy TD, Stuart JJ, Brown SJ, Beeman RW: Genetic linkage maps of the red hour beetle, Tribolium castaneum, based on bacterial artificial chromosomes and expressed sequence tags. Genetics 2005, 170(2):741-747.

30. McKay SD, Schnabel RD, Murdoch BM, Aerts J, Gill CA, Gao C, Li C, Matukumalli LK, Stothard P, Wang Z, et al: Construction of bovine wholegenome radiation hybrid and linkage maps using high-throughput genotyping. Animal Genetics 2007, 38(2):120-125.

31. Miles LG, Isberg SR, Glenn TC, Lance SL, Dalzell P, Thomson PC, Moran C: A genetic linkage map for the saltwater crocodile (Crocodylus porosus). Bmc Genomics 2009, 10.

32. Raudsepp T, Gustafson-Seabury A, Durkin K, Wagner ML, Goh G, Seabury CM, Brinkmeyer-Langford C, Lee EJ, Agarwala R, StallknechtRice $E$, et al: A 4,103 marker integrated physical and comparative map of the horse genome. Cytogenetic and Genome Research 2008, 122(1):28-36.

33. Remington DL, Whetten RW, Liu BH, O'Malley DM: Construction of an AFLP genetic map with nearly complete genome coverage in Pinus taeda. Theoretical and Applied Genetics 1999, 98(8):1279-1292.

34. Sanetra M, Henning F, Fukamachi S, Meyer A: A Microsatellite-Based Genetic Linkage Map of the Cichlid Fish, Astatotilapia burtoni (Teleostei): A Comparison of Genomic Architectures Among Rapidly Speciating Cichlids. Genetics 2009, 182(1):387-397.
35. Wang S, Zhang LL, Meyer E, Matz MV: Construction of a high-resolution genetic linkage map and comparative genome analysis for the reefbuilding coral Acropora millepora. Genome Biology 2009, 10(11).

36. Murphy WJ, Larkin DM, Everts-van der Wind A, Bourque G, Tesler G, Auvil L, Beever JE, Chowdhary BP, Galibert F, Gatzke L, et al: Dynamics of mammalian chromosome evolution inferred from multispecies comparative maps. Science 2005, 309(5734):613-617.

37. Roethele JB, Romero-Severson J, Feder JL: Evidence for broad-scale conservation of linkage map relationships between Rhagoletis pomonella (Diptera : Tephritidae) and Drosophila melanogaster (Diptera : Drosophilidae). Annals of the Entomological Society of America 2001, 94(6):936-947.

38. Wilfert L, Schmid-Hempel P, Gadau J: Bumblebee, Bombus terrestris. In Genome Mapping and Genomics in Animals. Volume 1. Edited by: Hunter W, Kole C. Berlin, Heidelberg: Springer Verlag; 2008:17-25.

39. Hoshiba H, Duchateau MJ, Velthuis HHW: Diploid Males in the Bumblebee Bombus terrestris (Hymenoptera), Karyotype Analyses of Diploid Females, Diploid Males and Haploid Males. Japanese Journal of Entomology 1995, 63(1):203-207.

40. Haberl M, Tautz D: Tri- and tetranucleotide microsatellite loci in honey bees (Apis mellifera) - a step towards quantitative genotyping. Molecular Ecology 1999, 8(8):1358-1360.

41. Solignac M, Vautrin D, Baudry E, Mougel F, Loiseau A, Cornuet JM: A microsatellite-based linkage map of the Honeybee, Apis mellifera $\mathrm{L}$. Genetics 2004, 167(1):253-262.

42. Solignac M, Vautrin D, Loiseau A, Mougel F, Baudry E, Estoup A, Garnery L, Haberl M, Cornuet JM: Five hundred and fifty microsatellite markers for the study of the honeybee (Apis mellifera L.) genome. Molecular Ecology Notes 2003, 3(2):307-311.

43. Van Oojen JW: JoinMap 4, Software for the calculation of genetic linkage maps in experimental populations.Edited by: Kyazma B.V. Wageningen; 2006.

44. Fishman L, Kelly AJ, Morgan E, Willis JH: A genetic map in the Mimulus guttatus species complex reveals transmission ratio distortion due to heterospecific interactions. Genetics 2001, 159(4):1701-1716.

45. Lange K, Boehnke M: How many polymorphic genes will it take to span the human genome. American Journal of Human Genetics 1982, 34(6):842-845.

46. Lattorff HMG, Moritz RFA: Recombination rate and AT-content show opposite correlations in mammalian and other animal genomes. Evolutionary Biology 2008, 35(2):146-149.

47. Gregory TR, Nicol JA, Tamm H, Kullman B, Kullman K, Leitch IJ, Murray BG, Kapraun DF, Greilhuber J, Bennett MD: Eukaryotic genome size databases. Nucleic Acids Research 2007, 35:D332-D338.

48. Lander ES, Green P, Abrahamson J, Barlow A, Daly MJ, Lincoln SE, Newburg L: Mapmaker - an interactive computer package for constructing primary genetic linkage maps of experimental and natural populations. Genomics 1987, 1(2):174-181.

49. Stam P: Construction of integrated genetic-linkage maps by means of a new computer package - Joinmap. Plant Journal 1993, 3(5):739-744.

50. Kosambi DD: The estimation of map distances from recombination values. Ann Eugenic 1943, 12:172-175.

51. Qi $X Q$, Stam P, Lindhout P: Comparison and integration of four barley genetic maps. Genome 1996, 39(2):379-394.

52. Liebhard R, Koller B, Gianfranceschi L, Gessler C: Creating a saturated reference map for the apple (Malus $\times$ domestica Borkh.) genome. Theoretical and Applied Genetics 2003, 106(8):1497-1508.

53. Mancera E, Bourgon R, Brozzi A, Huber W, Steinmetz LM: High-resolution mapping of meiotic crossovers and non-crossovers in yeast. Nature 2008, 454(7203):479-U471.

54. Zhao HY, Speed TP, McPeek MS: Statistical-analysis of crossover interference using the chi-square model. Genetics 1995, 139(2):1045-1056.

55. Button DK, Robertson BR: Determination of DNA content of aquatic bacteria by flow cytometry. Applied and Environmental Microbiology 2001, 67(4):1636-1645.

56. Dolezel J, Greilhuber J: Plant genome size estimation by flow cytometry: Inter-laboratory comparison. Annals of Botany 1998, 82:17-26.

57. Johnston JS, Bennett MD: Reference standards for determination of DNA content of plant nuclei. American Journal of Botany 1999, 86(5):609-613.

58. Bosco G, Campbell P: Analysis of drosophila species genome size and satellite DNA content reveals significant differences among strains as well as between species. Genetics 2007, 177(3):1277-1290. 
59. Wilfert L, Gadau J, Schmid-Hempel P: Variation in genomic recombination rates among animal taxa and the case of social insects. Heredity 2007, 98(4):189-197.

60. Nadeau JH, Taylor BA: Lengths of chromosomal segments conserved since divergence of man and mouse. Proceedings of the National Academy of Sciences of the United States of America-Biological Sciences 1984, 81(3):814-818.

61. Pevzner P, Tesler G: Genome Rearrangements in mammalian evolution: Lessons from human and mouse genomes. Genome Research 2003, 13(1):37-45

62. Lattorff HMG, Moritz RFA: Control of reproductive dominance by the thelytoky gene in honeybees. Biology Letters 2007, 3(3):292-295.

63. Beye M, Hasselmann M: The gene csd is the primary signal for sexual development in the honeybee and encodes an SR-type protein. Cell 2003, 114(4):419-429

64. Bourque G, Pevzner PA, Tesler G: Reconstructing the genomic architecture of ancestral mammals: Lessons from human, mouse, and rat genomes. Genome Research 2004, 14(4):507-516.

65. Faraut T: Addressing chromosome evolution in the whole-genome sequence era. Chromosome Research 2008, 16(1):5-16.

66. Stark A, Lin MF, Kheradpour P, Pedersen JS, Parts L, Carlson JW, Crosby MA, Rasmussen MD, Roy S, Deoras AN, et al: Discovery of functional elements in 12 Drosophila genomes using evolutionary signatures. Nature 2007, 450:219-232

67. Hoshiba H: Chromosome Evolution of Bees and Wasps (Hymenoptera, Apocrita) on the Basis of C-banding Pattern Analyses. Japanese Journal of Entomology 1993, 61(3):465-492.

68. Rascol VL, Pontarotti P, Levasseur A: Ancestral animal genomes reconstruction. Current Opinion in Immunology 2007, 19:542-546.

69. Cameron SA, Mardulyn P: Multiple molecular data sets suggest independent origins of highly eusocial behavior in bees (Hymenoptera: Apinae). Systematic Biology 2001, 50(2):194-214.

70. Danforth BN, Sipes S, Fang J, Brady SG: The history of early bee diversification based on five genes plus morphology. Proceedings of the National Academy of Sciences of the United States of America 2006, 103(41):15118-15123.

71. Engel MS: A giant honey bee from the Middle Miocene of Japan (Hymenoptera : Apidae). American Museum Novitates 2006, , 3504: 1-12.

72. Hines HM: Historical biogeography, divergence times, and diversification patterns of bumblebees (Hymenoptera : Apidae : Bombus). Systematic Biology 2008, 57(1):58-75.

73. Michener CD, Grimaldi DA: The oldest fossil bee - Apoid history, evolutionary stasis, and antiquity of social-behavior. Proceedings of the National Academy of Sciences of the United States of America 1988 85(17):6424-6426.

74. Whitfield JB, Kjer KM: Ancient rapid radiations of insects: Challenges for phylogenetic analysis. Annual Review of Entomology 2008, 53:449-472

75. Lidgard S, Crane PR: Quantitative analyses of the early angiosperm radiation. Nature 1988, 331(6154):344-346.

76. Wing SL: Evolution and Expansion of flowering plants. Paleontological Society Papers 2000, 6:209-231.

77. Clark AG, Eisen MB, Smith DR, Bergman CM, Oliver B, Markow TA, Kaufman TC, Kellis M, Gelbart W, lyer VN, et al: Evolution of genes and genomes on the Drosophila phylogeny. Nature 2007, 450(7167):203-218.

78. Chowdhary BP, Raudsepp T, Fronicke L, Scherthan H: Emerging patterns of comparative genome organization in some mammalian species as revealed by Zoo-FISH. Genome Research 1998, 8(6):577-589.

79. Everts van der Wind A, Larkin DM, Green CA, Elliott JS, Olmstead CA, Chiu R, Schein JE, Marra MA, Womack JE, Lewin HA: A high-resolution whole-genome cattle-human comparative map reveals details of mammalian chromosome evolution. Proceedings of the National Academy of Sciences of the United States of America 2005, 102(51):18526-18531.

80. Frönicke L, Wienberg J, Stone G, Adams L, Stanyon R: Towards the delineation of the ancestral eutherian genome organization: comparative genome maps of human and the African elephant (Loxodonta africana) generated by chromosome painting. Proceedings of the Royal Society of London Series B-Biological Sciences 2003, 270(1522):1331-1340.

81. Meyers SN, Rogatcheva MB, Larkin DM, Yerle M, Milan D, Hawken RJ, Schook LB, Beever JE: Piggy-BACing the human genome - II. A high- resolution, physically anchored, comparative map of the porcine autosomes. Genomics 2005, 86(6):739.

82. Milinkovitch MC, Helaers R, Depiereux E, Tzika AC, Gabaldon T: 2x genomes - depth does matter. Genome Biology 2010, 11(2).

83. Yang FT, Graphodatsky AS, Li TL, Fu BY, Dobigny G, Wang JH, Perelman PL, Serdukova NA, Su WT, O'Brien PCM, et al: Comparative genome maps of the pangolin, hedgehog, sloth, anteater and human revealed by crossspecies chromosome painting: further insight into the ancestral karyotype and genome evolution of eutherian mammals. Chromosome Research 2006, 14(3):283-296.

84. Stanyon R, Rocchi M, Capozzi O, Roberto R, Misceo D, Ventura M, Cardone MF, Bigoni F, Archidiacono N: Primate chromosome evolution: Ancestral karyotypes, marker order and neocentromeres. Chromosome Research 2008, 16(1):17-39.

85. Meznar ER, Gadau J, Koeniger N, Rueppell O: Comparative linkage mapping suggests a high recombination rate in all honeybees. J Hered 2010, 101(Suppl 1):S118-126.

86. Kuhl H, Beck A, Wozniak G, Canario AV, Volckaert FA, Reinhardt R: The European sea bass Dicentrarchus labrax genome puzzle: comparative BAC-mapping and low coverage shotgun sequencing. BMC Genomics 2010, 11(1):68

87. MISA: - MIcroSAtellite identification tool., [pgrcipk-gaterslebende/misa/]

88. Hall TA: BioEdit: a user-friendly biological sequence alignment editor and analysis program for Windows 95/98/NT. Nucleic Acids Symposium Series 1999, 41:95-98.

89. Katoh K, Kuma K, Toh H, Miyata T: MAFFT version 5: improvement in accuracy of multiple sequence alignment. Nucleic Acids Research 2005, 33(2):511-518.

90. You FM, Huo NX, Gu YQ, Luo MC, Ma YQ, Hane D, Lazo GR, Dvorak J, Anderson OD: BatchPrimer3: A high throughput web application for PCR and sequencing primer design. BMC Bioinformatics 2008, 9.

91. Untergasser A, Nijveen H, Rao X, Bisseling T, Geurts R, Leunissen JAM: Primer3Plus, an enhanced web interface to Primer3. Nucleic Acids Research 2007, 35:W71-W74.

92. Murray TE, Fitzpatrick U, Brown MJF, Paxton RJ: Cryptic species diversity in a widespread bumblebee complex revealed using mitochondrial DNA RFLPs. Conservation Genetics 2008, 9(3):653-666.

93. Voorrips RE: MapChart: Software for the graphical presentation of linkage maps and QTLs. Journal of Heredity 2002, 93(1):77-78.

94. Chakravarti A: A graphical representation of genetic and physical maps the Marey map. Genomics 1991, 11(1):219-222.

doi:10.1186/1471-2164-12-48

Cite this article as: Stolle et al:: A second generation genetic map of the bumblebee Bombus terrestris (Linnaeus, 1758) reveals slow genome and chromosome evolution in the Apidae. BMC Genomics 2011 12:48.

\section{Submit your next manuscript to BioMed Central and take full advantage of:}

- Convenient online submission

- Thorough peer review

- No space constraints or color figure charges

- Immediate publication on acceptance

- Inclusion in PubMed, CAS, Scopus and Google Scholar

- Research which is freely available for redistribution

Submit your manuscript at www.biomedcentral.com/submit
C Biomed Central 